\title{
Management of bronchopulmonary neuroendocrine tumors
}

\author{
Garima Gupta, MD ${ }^{1}$ (D), Robert A Ramirez, DO² (D, Aman Chauhan, MD ${ }^{1}$ (D) \\ ${ }^{1}$ Markey Cancer Center, University of Kentucky, ${ }^{2}$ Division of Hematology/Oncology, Vanderbilt University Medical Center \\ Keywords: neuroendocrine tumors, bronchopulmonary, typical carcinoid, atypical carcinoid \\ https://doi.org/10.53876/001c.32244
}

\section{International Journal of Cancer Care and Delivery}

Vol. 2, Issue 1, 2022

\begin{abstract}
Bronchopulmonary neuroendocrine tumors (BPNETs) are the second most common subset of NETs after gastroenteropancreatic NETs. Historically, most clinical studies have excluded BPNETs during drug development and the data is often extrapolated from non-BPNETs. However, growing burden of BPNETs and the development of novel treatment strategies including targeted therapies and radiopharmaceuticals have paved the way for revisiting treatment strategies. In this review, we go over recent advances in the management of BPNETs and summarize consensus guidelines with the help of research data and clinical scenarios.
\end{abstract}

\section{BACKGROUND}

Neuroendocrine tumors (NETs) arise from enterochromaffin or Kulchitsky cells which are widely dispersed throughout the body and thus, can form in different locations such as the gastrointestinal (GI) tract, pancreas, and lungs. The incidence of NETs has been on the rise with bronchopulmonary NETs (BPNETs) being the second most common, comprising $20-30 \%$ of all NETs. ${ }^{1,2}$ This trend is likely attributed to increased awareness of NETs, improved radiographic technology that is more widely available, and increased screening for cancers. Based on the World Health Organization (WHO) 2015 classification, the four histologic variants of lung NETs include small cell lung carcinoma (SCLC), large cell neuroendocrine carcinoma (LCNEC), and carcinoid tumors which are further divided into typical carcinoid (TC) and atypical carcinoid (AC) tumors. ${ }^{3,4}$ For this review, BPNETs will refer to TC and AC tumors.

While they fall under the umbrella of neuroendocrine neoplasms, SCLC and LCNEC are collectively called poorly differentiated high-grade neuroendocrine carcinomas, significantly different from well-differentiated low-grade TC and intermediate-grade AC tumors in terms of their clinical behavior and epidemiological, genetic, and molecular findings. ${ }^{5}$ BPNETs are often diagnosed in patients between 40-60 years of age and without a history of smoking, suggesting different underlying biology and etiological factors compared to other lung cancers. ${ }^{2}$ Besides various cytological and histologic characteristics including the extent of necrosis, Ki-67 nuclear expression is useful in separating high-grade SCLC and LCNEC from BPNETs. ${ }^{3}$ For further identification, the mitotic count is key in differentiating TC from AC tumors and should be performed per $2 \mathrm{~mm}^{2}$ in the areas of highest activity. ${ }^{3,4}$ While TC tumors are characterized by less than 2 mitoses $/ 2 \mathrm{~mm}^{2}$ and absence of necrosis, AC tumors have $2-10$ mitoses $/ 2 \mathrm{~mm}^{2}$ with presence/absence of necrosis. These findings are summarized in Table 1 .
The WHO classification also recognizes diffuse idiopathic pulmonary neuroendocrine cell hyperplasia (DIP$\mathrm{NECH}$ ) as a preinvasive lesion. DIPNECH is rare, manifested as hyperplasia of neuroendocrine cells which may be diffuse, or in rows or clusters, and is usually confined to the bronchial or bronchiolar epithelium. When proliferation of neuroendocrine cells extends beyond the epithelium, crossing the basement membrane into the stroma, it forms tumorlets. These lesions are often discovered incidentally as peribronchiolar nodular aggregates and are morphologically identical to TC tumors but measure less than $5 \mathrm{~mm}$ in size. ${ }^{6}$ DIPNECH and tumorlets can co-concurrently be seen with BPNETs.

Up to $64 \%$ of BPNETs are located centrally with a higher rate noted in TC tumors in some studies, which makes patients more prone to obstructive respiratory symptoms compared to those with peripherally located BPNETs, which are often discovered incidentally. ${ }^{7-9}$ Patients can present with symptoms such as coughing, hemoptysis, wheezing, chest pain, and recurrent pneumonia, and they are sometimes repeatedly treated for common respiratory diseases such as chronic obstructive pulmonary disease and asthma until another pathological process is suspected, leading to a delay in diagnosis.

In about $90 \%$ of the cases, BPNETs are diagnosed as solitary lesions. ${ }^{7}$ Variable rates of lymph node involvement at the time of diagnosis have been reported. Overall, a higher rate is observed in AC tumors, with one study reporting lymph node involvement in $57 \%$ of patients with AC compared to $14 \%$ in patients with TC tumors. ${ }^{7,10-12} \mathrm{Be}-$ sides intrathoracic spread, the most common sites of distant metastasis are bone and liver, noted in about $20 \%$ of patients with AC and 3\% with TC tumors. ${ }^{2,11}$ Metastasis to the central nervous system has been reported as well and was seen in patients with AC tumors. ${ }^{13}$

In $2-5 \%$ of cases, the tumor may be functional and present with carcinoid syndrome. This has been noted especially in the presence of liver metastases but is less common 
Table 1. 2021 WHO Pathological Classification of Lung Carcinoids

\begin{tabular}{llll}
\hline & Grade & ${ }^{*}$ Mitoses $/ 2 \mathrm{~mm}^{2}$ & Necrosis \\
\hline Typical Carcinoid & $\mathrm{I}$ & $<2$ & Absent \\
\hline Atypical Carcinoid & $\mathrm{II}$ & $2-10$ & Absent/Present \\
\hline
\end{tabular}

Note: Defining criteria for BPNETs include organoid growth patterns, absent to focal/punctate comedo-like necrosis, mitoses up to 10 per $2 \mathrm{~mm}^{2}$ and positive immunohistochemistry for neuroendocrine markers.

"In tumors that are near the cutoffs for mitoses, at least three sets of $2 \mathrm{~mm}^{2}$ should be counted and the mean used for determining the mitotic rate, rather than the single highest rate

Abbreviations: BPNET, Bronchopulmonary Neuroendocrine Tumor

in BPNETs compared to patients with GI NETs. ${ }^{11,14,15}$ Cushing's syndrome is also rarely found, however, up to $40 \%$ of patients with ectopic Cushing's have been reported to have a BPNET. ${ }^{16}$ Other rare presentations that have been reported include acromegaly and hypoglycemia secondary to tumors secreting growth hormone-releasing hormone and insulin-growth factor $2 .{ }^{17,18}$ There is limited utility of routine baseline plasma chromogranin A ( CgA) testing in patients with BPNETs and poor correlation with disease status. ${ }^{19} \mathrm{CgA}$ can also be falsely elevated with renal impairment, atrophic gastritis, and the use of proton pump inhibitors. ${ }^{20}$ Other biochemical tests such as histamine, urine 5-hydroxyindoleacetic acid (HIAA), cortisol, and adrenocorticotropic hormone (ACTH) should be obtained as clinically indicated.

The initial gold standard radiological test is contrast-enhanced computed tomography (CT), which identifies both the primary lesion and intrathoracic lymphadenopathy. BPNETs usually range in size from 2-5 cm and may either be purely intraluminal appearing as a polypoid lesion or purely extraluminal. More frequently though, they are partially intraluminal with an extraluminal component, referred to as iceberg morphology. ${ }^{21,22}$ Since DIPNECH primarily affects the bronchial wall, it presents with CT changes characteristic of airway-related diseases. Bilateral mosaic attenuation, air trapping, and multifocal micronodules on expiratory CT have been reported. ${ }^{23}$ Multiphase CT or magnetic resonance imaging (MRI) of the abdomen should also be used to evaluate for metastasis to the liver and bone.

The role of positron emission tomography (PET)/CT in the diagnosis of NETs has evolved significantly over the years. NETs are known to overexpress somatostatin receptors (SSTRs), which provides a molecular basis for functional imaging and therapeutic application of somatostatin analogs (SSAs). In one study, investigators studied the expression of SSTR1 to SSTR5 in 178 BPNETs and found that $75 \%$ of tumors expressed SSTR2. ${ }^{24}$ Therefore, besides Fluorine 18-fluorodeoxyglucose $\left({ }^{18} \mathrm{~F}-\mathrm{FDG}\right)$, SSAs such as DOTANOC, DOTATOC, DOTATATE labeled with ${ }^{68} \mathrm{Ga}$ and ${ }^{64} \mathrm{Cu}$ are now widely used as part of the standard diagnostic workup. ${ }^{68} \mathrm{Ga}$ and ${ }^{64} \mathrm{Cu}$-DOTATATE are now both approved by the Food and Drug Administration (FDA) and available for diagnostic purposes in the US. When available, these are preferred over somatostatin receptor scintigraphy (SRS) due to higher sensitivity. ${ }^{25}$ Not surprisingly, PET/CT using ${ }^{68} \mathrm{Ga}$-DOTATOC has been reported to be superior to ${ }^{18} \mathrm{~F}$ FDG in diagnoses of TC. The reverse has been observed in patients with AC. ${ }^{26,27}$ This is because AC tumors have a higher grade than TC tumors and are, therefore, hypermetabolic and more aggressive. The degree of uptake on ${ }^{8} \mathrm{~F}$ FDG PET/CT can help provide prognostic information and uptake on PET/CT with somatostatin analogs is a prerequisite for peptide receptor radionuclide therapy (PRRT). ${ }^{28}$

To obtain a histologic diagnosis, bronchoscopy for central tumors and either an endoscopic transbronchial or CTguided transthoracic biopsy for peripheral tumors is performed. When there is evidence of distant metastasis, a metastatic lesion easier to access may be biopsied instead of the primary lesion. Since there is not a staging system that has been designed specifically for BPNETs, the TumorNode-Metastasis (TNM) model for lung cancers in the $8^{\text {th }}$ edition of the American Joint Committee on Cancer (AJCC) is used. ${ }^{29}$

The overall 5-year survival rate of patients with BPNETs has been reported to be $73.5 \%$ with rates as high as $92 \%$ reported in patients with TC tumors. ${ }^{2,9,11,12}$ Nodal involvement and presence of distant metastases are independently associated with a negative impact on survival. ${ }^{30,31}$ Absence of nodal involvement, referred to as N0 disease, confers excellent prognosis compared to patients with N1 or N2 disease with 5-year survival rates as high as $97 \% .9,10$ While not validated by the WHO for BPNETs, Ki-67 was found to be an independent prognostic marker in one study. ${ }^{31}$

Treatment of BPNETs requires a multidisciplinary approach and given their rarity, referral to a tertiary academic NET center is preferred. There are several factors to be considered in optimal management of BPNETs such as tumor size, location, presence of nodal/distant metastasis, comorbidities, presence of hormonal symptoms, and previous lines of therapy. For patients with metastatic disease, an assessment of the tumor growth rate based on serial imaging is important. Systemic treatment is reserved for when there is clinical or radiographic progression, uncontrolled or worsening symptoms or if there is high disease burden, especially in the liver. In this review, we summarize current literature including guidelines from North American Neuroendocrine Tumor Society (NANETS), The European Neuroendocrine Tumor Society (ENETS), and National Comprehensive Cancer Network (NCCN), and provide updates on recent developments in the management of $\mathrm{BP}$ NETs. 


\section{MANAGEMENT}

\section{SURGERY AND ADJUVANT TREATMENT}

Surgical resection, when feasible, remains the initial treatment of choice for localized disease and provides a chance of cure. The choice of technique and extent of surgical resection depends on tumor location, size, pre-operative degree of suspicion for nodal involvement, and whether it is a TC or an AC tumor. Pre-operative histologic diagnosis is usually known, however, if only a small specimen was obtained during initial biopsy, it may become challenging to differentiate between TC and AC tumors. ${ }^{10,32}$ In some cases, patients are taken directly to surgery or rarely, these tumors may be incidentally discovered on surgical specimens post-operatively. Overall, the guiding principles for surgical resection of non-small cell lung cancer (NSCLC) are applied for BPNETs with lobectomy being the gold standard, however with special considerations due to their low grade and indolent clinical behavior. Minimally invasive and parenchymal sparing techniques have become increasingly preferable when possible. The choice of lung-sparing surgical technique is often dependent on the institution and surgeon's training and experience.

For peripherally located TC tumors measuring less than $2 \mathrm{~cm}$, lung-sparing surgery is an option if an R0 resection can be achieved. ${ }^{33}$ However, for larger peripheral tumors and when there is concern for $\mathrm{AC}$, patients with adequate pulmonary reserve should be considered for a lobectomy. ${ }^{34}$ Similarly, for centrally located tumors, most of which are TC, parenchymal-sparing resections including bronchoplastic procedures, bronchial sleeve, or sleeve lobectomy are preferred over pneumonectomy or bilobectomy. In studies with patients with N0 TC tumors, lobectomy versus sublobar resection yielded similar 5-year overall survival (OS) rates. ${ }^{35,36}$ In the same population, a clear survival advantage was noted for patients who underwent surgery versus observation. However, the 5-year disease-specific survival was still high at $88 \%$ in patients who underwent observation-only, suggesting it may be reasonable to consider this approach in patients who are at high risk for surgery-related morbidity and mortality. ${ }^{36}$ In one study, due to a high rate of lymph node involvement and multicentric forms noted in their patients, authors recommended an aggressive approach with lobectomy and lymph node dissection for all patients. ${ }^{10}$ Typically, a $5 \mathrm{~mm}$ negative margin is considered appropriate.

Lymph node upstaging has been found to be a strong independent predictor of OS. ${ }^{35}$ Systemic hilar and mediastinal lymph node dissection is, therefore, highly advocated in patients due to this reason and high rates of nodal involvement, especially in patients with AC tumors. Pre-operative mediastinal staging should especially be considered in patients with clinical concern for N1 or N2 disease or peripherally located N0 tumors but with suspicion for AC. ${ }^{34}$

In cases of intraluminal BPNETs without an extraluminal component, endobronchial mechanical or laser-assisted resection can be considered. ${ }^{37,38}$ In one study, patients with purely intraluminal BPNETs measuring less than $20 \mathrm{~mm}$ in diameter on CT were noted to be good candidates despite histological grade with procedure-associated bleeding in $9 \%$ of patients. Interestingly, endobronchial treatment was also successfully performed in $28 \%$ of patients with possible extraluminal disease noted on CT. ${ }^{39}$ In a small study, cryotherapy was performed as adjunctive treatment to endobronchial resection and was noted to be safe and effective with only $1 / 18$ patients noted to have recurrence after seven years. In another study, presurgical endoscopic resection of large tissues specimens performed in nine patients allowed for more accurate histological diagnosis and assessment of tumor base and improvement in respiratory status with potential impact on the ability to perform a lung-sparing surgery. ${ }^{40}$

There are no large prospective studies to evaluate the role of adjuvant therapy for BPNETs. The NCCN, NANETS, and European Society of Medical Oncology (ESMO) have varying recommendations for adjuvant chemotherapy of BPNETs. NCCN recommends against adjuvant treatment for stage I and II BPNETs but recommends considering it for stage IIIA patients based on expert opinion. Specifically, they recommend considering either a platinum doublet (cisplatin/carboplatin + etoposide) or temozolomide for patients with stage IIIA disease with either intermediate grade and negative margins or positive margins irrespective of histology. However, they also state the lack of data to support this recommendation. ${ }^{41}$ Chemotherapy may also be combined with radiation if considered appropriate. ESMO recommends the consideration of adjuvant treatment for patients with AC and nodal disease. NANETS recommends against adjuvant treatment. ${ }^{33,42}$ Current literature does not suggest a consistent benefit in OS with adjuvant chemotherapy. ${ }^{43}$ Some authors have recommended its use in a subset of patients with adverse pathologic features and lymph node involvement based on a trend towards improved OS compared to published literature, however, this evidence is limited. ${ }^{44,45}$ In fact, in one study, in patients with TC tumors, adjuvant treatment was associated with significantly worse outcomes ${ }^{46}$ and should, therefore, not be used outside of a clinical trial.

For patients who are not surgical candidates or have unresectable locally advanced BPNET, NCCN recommendations are based on tumor grade. ${ }^{41}$ For intermediate grade stage III A/B/C BPNETs, platinum-doublet chemotherapy with or without radiation may be considered. For low-grade stage III A/B/C BPNETs, observation versus other systemic therapy options discussed under medical management below may be considered. If patients with intermediate-grade stage III A/B/C BPNETs are poor candidates for aggressive treatment with chemotherapy, other systemic therapies may be used with or without radiation. SSA are overall welltolerated and can manage symptoms and also provide an anti-proliferative effect. Radiation alone may be used for palliation if systemic therapy is contraindicated.

\section{MEDICAL MANAGEMENT}

The goal of systemic therapy in the treatment of NETs includes control of both symptoms and tumor growth. Systemic treatment options for BPNETs include SSAs, targeted 
therapies, chemotherapies, PRRT, and clinical trials. These treatment options are used for patients with unresectable or metastatic disease. The sequencing of these treatment options is not well defined. An individualized patient-centered approach based on clinical status and radiological findings is essential in formulating an optimal treatment plan.

\section{A) SOMATOSTATIN ANALOG THERAPY}

Treatment with SSAs including lanreotide autogel (LAN) and octreotide long-acting release (LAR) is well tolerated and effectively manages symptoms as well as provides antiproliferative effect in patients with NETs. SSAs are recommended by NANETS, ENETS, and NCCN in the management of BPNETs. The PROMID and CLARINET study established SSAs as first-line monotherapy in patients with NETs of the GI tract and pancreas. ${ }^{47,48}$ In the PROMID study, 85 patients with well-differentiated metastatic midgut NETs received either placebo or octreotide LAR $30 \mathrm{mg}$ every month. There was a statistically significant improvement in median time to tumor progression (TTP) of 6 months in placebo versus 14.3 months in the treatment arm. ${ }^{48}$ In the CLARINET study, 204 patients with advanced gastroenteropancreatic (GEP) NETs received either placebo or LAN $120 \mathrm{mg}$ every 28 days. There was a statistically significant improvement in progression-free survival (PFS) with median PFS not yet reached at the time of publication in the treatment arm compared to 18 months in the placebo arm. ${ }^{47}$ Results of the open-label extension of the CLARINET study revealed a median PFS of 38.5 months. ${ }^{49}$ None of these studies, however, included patients with BPNETs.

Three retrospective studies including patients with BPNETs who received SSAs either as first ${ }^{50,51}$ or first/subsequent $^{52}$ line therapy have been published. The initial study that described the use of SSAs in first-line setting in BPNETs only was published in 2017, included 20 patients treated with octreotide LAR and 10 patients with lanreotide. Twenty-three out of 30 patients had AC tumors. Stable disease (SD) was observed in $87 \%$ of patients and partial response (PR) in one patient. The 5-year survival rate was $53 \%$ and the median PFS was 11.1 months. ${ }^{50}$ Another recent study published in 2021 included 31 patients with a fairly equal number of AC (17) and TC (14) tumors. This study demonstrated SD in $77.4 \%$ of patients with a median PFS of 28.6 months. ${ }^{51}$ The largest of these studies included 61 patients, $76 \%$ of whom received SSAs as first-line therapy. In this study, the median PFS was 17.4 months and the median OS was 58.4 months. ${ }^{52}$

Most recently, the findings from the phase III SPINET trial were presented at the 2021 NANETS symposium. This trial randomized patients with BPNETs to either LAN plus best supportive care versus placebo. Enrollment to the trial was stopped early due to slow accrual. A total of 77 patients were randomized and treated. In this trial, the median PFS was 16.6 months (95\% CI, 11.3 to 21.9 ) in the treatment arm versus 13.6 months (95\% CI, 8.3 to not calculable) in the placebo arm with a hazard ratio (HR) of 0.90 (95\% CI, 0.46-1.88). Specifically, the median PFS was 21.9 months with treatment versus 13.9 months with placebo in patients with TC tumors and 13.8 months with treatment versus 11 months with placebo in patients with AC tumors. ${ }^{53}$

\section{B) TARGETED THERAPIES}

Targeted agents that have been studied in the treatment of BPNETs include everolimus, an oral inhibitor of the mammalian target of rapamycin (mTOR) pathway, and antiangiogenics such as sunitinib and pazopanib. Out of these, everolimus has the highest quality evidence to support its use in the treatment of BPNETs and was approved by the FDA in 2016 in this setting.

\section{1) EVEROLIMUS}

mTOR is a serine/threonine kinase, downstream of the phosphatidylinositol-3-kinase (PI3K)/Akt pathway, activation of which results in significant disturbance in controlling cell growth and survival. The role of this pathway in tumorigenesis, specific to NETs, has been demonstrated. ${ }^{54,55}$ Everolimus is a derivative of rapamycin and an inhibitor of this pathway. The pre-clinical data with human BPNETs in primary cultures treated with everolimus showed inhibition of mTOR complex 1 (mTORC1) and thus, was a promising therapeutic target. ${ }^{56}$ Everolimus was first approved in the treatment of advanced, progressive pancreatic NETs based on phase III data from the RADIANT-3 trial, published in 2011, which showed a median PFS of 11 months in patients who received everolimus at $10 \mathrm{mg}$ daily versus 4.6 months with placebo. ${ }^{57}$ Matured data showed a non-statistically significant survival benefit of 6.3 months, which was attributed to a large crossover from the placebo arm. ${ }^{58}$

Shortly thereafter, the RADIANT-2 trial was published, which included patients with advanced NETs from any primary site, associated with carcinoid syndrome. Patients received either everolimus or placebo, both in conjunction with octreotide LAR monthly. The median PFS in the treatment arm was 16.4 months (95\% CI, 13.7 to 21.2) compared to 11.3 months (95\% CI, 8.4 to 14.6 ) in patients who received placebo and SSA with an HR of 0.77 (95\% CI 0.59 to $1.00 ; p=0.026) .{ }^{59}$ However, the final OS analysis was not consistent with these initial findings. The median OS was 29.2 months in the treatment arm versus 35.2 months in the placebo arm with an HR of 1.17 .60 Several factors were speculated to influence these results including heterogeneous study population, crossover from the placebo group, unequal SSA use, and some patients who may not have needed to start aggressive therapy, which in turn may have led to more frequent adverse events. This study emphasized the importance of the "wait and watch" strategy as some patients may have indolent disease for years and could be monitored closely, reserving treatment options for time of progression. A sub-group analysis of the RADIANT-2 trial including 44 patients with BPNETs only was later published. Thirty-three of these patients had received everolimus and the median PFS was 13.63 months (95\% CI, 5.55 to 14.29 ) in patients who received everolimus plus SSA versus 5.59 months ( $95 \%$ CI, 2.79 to 27.76 ) in placebo plus SSA, a 2.4 fold improvement, with an HR of 0.72 (95\% CI, $0.31-1.68 ; p=0.228$ ). The authors also noted that $67 \%$ 
of the patients who received everolimus had minor tumor shrinkage compared to $27 \%$ in the placebo arm. ${ }^{61}$

Following this, results of the RADIANT- 4 trial were published. This international trial enrolled 302 patients with advanced, progressive, well-differentiated, and non-functional (unlike RADIANT-2) NETs originating in the GI tract or lungs to receive either everolimus or placebo. The median PFS in the treatment arm was 11 months compared to 3.9 months in the placebo group with an HR of 0.48 . Treatment was well-tolerated with infrequent grade 3 or 4 drugrelated events, which included stomatitis, diarrhea, anemia, fatigue, and hyperglycemia. ${ }^{62}$ A post hoc analysis of the lung subgroup with 90 patients showed a median PFS of 9.2 months in the everolimus arm versus 3.6 months in the placebo arm with an HR of 0.5 .63

To further study the role of the everolimus in treatmentnaïve patients and its combination with SSA due to possible synergy secondary to their effects on the mTOR pathway, the ITMO trial was designed. This was a phase II study that enrolled 50 patients with NETs of multiple origins including 11 patients with BPNETs and received everolimus in combination with octreotide LAR. The overall response rate (ORR) was $18 \%$ with SD noted in $74 \%$ of patients. ${ }^{64}$ A 5-year update showed promising results with a median TTP of 33.6 months and the median OS of 61 months. ${ }^{65}$ The phase II LUNA trial was the only study to enroll patients with advanced, well-differentiated thymic and BPNETs only and had three separate arms, pasireotide alone, everolimus alone, or a combination of both. The median PFS was 8.5, 12.5 , and 11.8 months in the pasireotide, everolimus, and combination group, respectively. 66

The NCCN and ENETS guidelines recommend using everolimus in the first-line setting in patients with BPNETs who have progressive disease or high-tumor burden. Everolimus may be combined with SSAs, especially in patients with functional BPNETs. The initial dose is $10 \mathrm{mg}$ and is typically reduced in increments of $5 \mathrm{mg}$ or less depending on tolerability. Patients may develop resistance and while previous studies have identified resistance mechanisms, no agents with promising safety profiles have been developed to overcome these resistance pathways so far. ${ }^{54}$

\section{2) ANTIANGIOGENICS}

The data for the use of antiangiogenics in the treatments of BPNETs is not as robust. Sunitinib is used in the treatment of pancreatic NETs based on prospective trials ${ }^{67}$ but the results for BPNETs have not been impressive. In a phase II trial, sunitinib was prescribed at a dose of $50 \mathrm{mg} /$ day for 4 weeks on followed by 2 weeks off. The trial enrolled 109 patients, including 14 patients with gastric and BPNETs. Among these patients, while the ORR was only $2.4 \%, 83 \%$ of patients had SD with 10.2 months of median TTP. ${ }^{68}$ In the phase II PAZONET study, a total of 44 patients with advanced NETs who had failed on other systemic therapies were enrolled, 5 of whom had BPNETs. The shorter median PFS of 3.4 months was noted in patients with lung and thymic NETs compared to 12.8 months in pancreatic NETs and 10 months in GI NETs. ${ }^{69}$ A combination of sorafenib and bevacizumab has been used but yielded unfavorable safety results. ${ }^{70}$ Bevacizumab has also been compared to pegylated (PEG) interferon alpha-2b, both used in combination with SSA in a phase II study. A total of 44 patients were enrolled including four with BPNETs. A higher PFS rate of $85 \%$ after 18 weeks of treatment was observed in the bevacizumab arm compared to $68 \%$ in the PEG interferon alpha-2b arm. ${ }^{71}$ Recently, in a phase III study, 198 patients with advanced extrapancreatic NETs, including 23 patients with BPNETs, were treated with either surufatinib or placebo. The median PFS in the treatment arm was 9.2 months versus 3.8 months in the placebo arm. ${ }^{72}$

\section{C) CHEMOTHERAPY}

Systemic chemotherapy is typically reserved for patients with metastatic and progressive BPNETs. Data for several different regimens has evolved over the years but needs to be interpreted with caution due to the often mixed population of primary tumors and the small number of patients with BPNETs in studies.

Initial studies dating back to 2001 demonstrated discouraging results. In a study with 31 patients with metastatic BPNETs, 7 of 7 patients progressed on streptozotocin and 5-fluorouracil (5-FU), while SD for up to 10 months was achieved in 2 of 2 patients treated with streptozotocin and doxorubicin. ${ }^{73}$ Subsequently, a phase II/III trial with 249 patients with advanced NETs including 22 patients with BPNETs compared 5-FU/doxorubicin with 5-FU/streptozotocin. There were no differences between 5 -FU/doxorubicin and 5-FU/streptozotocin in terms of response rates and median PFS, however, 5-FU/streptozotocin was noted to be superior in median survival. Patients who progressed on either regimen crossed over to dacarbazine and a response rate of $8.2 \%$ was noted. ${ }^{74}$ Following this, 5-FU/streptozotocin was also combined with cisplatin and this combination resulted in a $25 \%$ response rate for non-pancreatic primary sites. ${ }^{75}$ In a subsequent trial, however, no added benefit of cisplatin to capecitabine/streptozotocin was noted. ${ }^{76}$ A patient with metastatic BPNET was also successfully treated with a combination of liposomal doxorubicin and capecitabine after progressing on cisplatin and etoposide. These combinations are not routinely used in practice for BPNETs. ${ }^{77}$

The platinum doublet combination used in the first-line setting for LCNEC and SCLC has shown to be beneficial only in a subset of patients with BPNETs, most likely secondary to their less aggressive biology and lower grade. ${ }^{78}$ In a retrospective analysis, 13 patients with metastatic BPNETs treated with etoposide and platinum combination showed a response rate of $23 \%$, a disease control rate (DCR) of $57 \%$, and a median PFS of 7 months. ${ }^{44}$ Similar responses were noted in another retrospective study where $23.5 \%$ of patients with metastatic BPNETs treated with etoposide and platinum combination were noted to have responses and had a median PFS of 7 months. ${ }^{13}$

Other platinum-based regimens such as 5-FU, leucovorin and oxaliplatin (FOLFOX), capecitabine and oxaliplatin (CAPOX), and gemcitabine and oxaliplatin (GEMOX) have also shown efficacy. In one retrospective analysis, 31 patients with grade $1 / 2$ NETs, including 8 patients with BP- 
NETs treated with one of these oxaliplatin-based regimens, were studied. The overall DCR was $70 \%$ and the median PFS was 14.1 months with no significant differences between Ki-67 subgroups of $<5 \%$ and $5-20 \% .{ }^{79,80}$ CAPOX was studied in 24 patients with metastatic NETs, $63 \%$ of whom were classified as grade $1 / 2$ and $17 \%$ originated in the lungs. Out of these, $29 \%$ of patients achieved a PR and the median TTP was 9.8 months. ${ }^{81}$ In a phase II trial, 40 patients with advanced NETs were enrolled and treated with CAPOX, 27 of whom had well-differentiated tumors and 10 patients had NETs originating in the lungs. This regimen was found to be effective and was well tolerated. ${ }^{82}$ Among these, for patients with well-differentiated NETs, 30\% had a PR and 38\% had SD. In one study with BPNETs only, either GEMOX or FOLFOX was used in 45 patients including both treatment naïve and pre-treated patients, and showed similar outcomes with either regimen. The median PFS was 15 months and the median OS was 34 months. ${ }^{83}$ In a larger study with 78 patients with mainly grade 2 NETs, 19 of whom had BPNETs, patients received either CAPOX, GEMOX, or FOLFOX. The median PFS was 8 months and the median OS was 32 months. ${ }^{80}$ Single-agent metronomic 5 -FU with octreotide LAR has also shown efficacy in a phase II trial, however, no patients with BPNETs were included in this study. ${ }^{84}$

Multiple studies with temozolomide both as monotherapy and in combination with other agents such as SSAs, bevacizumab, and capecitabine have been published. In a retrospective analysis of 31 patients treated with temozolomide, PR was noted in $14 \%$ and SD in $52 \%$ of patients. The median PFS was 5.3 months and the median OS was 23.3 months. ${ }^{85}$ Recently, results of ATLANT phase II trial were published, where 40 patients with progressive BPNETs and thymic NETs were enrolled and treated with LAN and temozolomide for 12 months. At 9 months, the DCR was 35\% and the median PFS was 5.3 months. ${ }^{86}$ In a phase II study, temozolomide was combined with bevacizumab in patients with pancreatic and extra-pancreatic NETs, including 4 patients with BPNETs. Response rates were higher in pancreatic NETs with no PRs noted in extra-pancreatic NETs. However, SD was achieved in $74 \%$ of the patients and the median PFS was 7 months. ${ }^{87}$ Multiple studies with combination of capecitabine and temozolomide (CAPTEM) have shown promising results. Several retrospective analyses have reported a DCR of over $70 \% .{ }^{88-91}$ In one study, the median PFS and OS were 13 and 68 months, respectively. ${ }^{91}$ A trend of better outcomes in patients with lower Ki-67 values was noted. 88,90 MGMT methylation was associated with higher disease control and prolonged TTP. ${ }^{89}$ Interim analysis of a phase II study of patients with well-differentiated NETs treated with CAPTEM showed a median PFS $>22$ months. ${ }^{92}$

\section{D) PEPTIDE RECEPTOR RADIONUCLIDE THERAPY (PRRT)}

PRRT may be used in patients with metastatic BPNETs who have positive uptake on SSTR imaging, either PET/CT or SRS. High energy beta-emitting radionuclides, Ytrrium-90 (Y-90) or Lutetium-177 (Lu-177) are bound to SSAs such as DOTATOC and DOTATATE to reach and penetrate SSTR positive cells. The goal of treatment is disease control and palliation of symptoms. In a phase II study with 41 patients including 7 with BPNETs, 100\% of patients with BPNETs achieved disease control with no progressive disease within the follow-up period. ${ }^{93}$ Subsequently, in a large phase II study with 1109 mixed population of NET patients, disease control was achieved in $39 \%$ of patients with a median survival of 94.6 months. In 84 patients with BPNETs, a morphologic response was noted in $28.6 \%$ of patients and clinical response in 38\%. Tumoral uptake in the initial imaging study was an independent predictor of OS. ${ }^{94}$

In a retrospective study with 135 patients, $13 \%$ of whom had BPNETs revealed a median OS of 40 months from the date of first PRRT. Specifically, in patients with BPNETs, the median OS from first PRRT was 32.4 months and TTP was 18.6 months. ${ }^{95}$ In another retrospective study compromising of 114 patients with BPNETs treated with PRRT, the median PFS was 28 months and the median OS 58.8 months. ${ }^{96}$ Other retrospective analyses of patients with BPNETs have also revealed a median OS ranging between 40-42 months. Intense uptake (SUVmax > 5) on ${ }^{18}$ F-FDG PET/CT was associated with a poor response ${ }^{97}$ and high hepatic tumor burden and high CgA levels were noted to be negative predictors of survival. 98

While it didn't include patients with BPNETs, the results of the NETTER-1 trial are worth noting as they could potentially be extrapolated to other NETs. This trial enrolled patients with inoperable well-differentiated midgut NETs to receive either PRRT or octreotide LAR and showed a substantial prolongation of PFS in the PRRT arm. ${ }^{99}$ Matured data was recently published and showed a non-significant improvement in median OS to 48 months in the PRRT arm versus 36.3 months in the control arm, potentially due to large cross-over. ${ }^{100}$ A phase II trial that aims to assess PFS in patients with advanced or metastatic BPNETs treated with either PRRT or everolimus is currently underway [NCT04665739].

Patients need to be monitored for hematological and renal toxicities that are usually mild and transient but may sometimes result in permanent damage. In one study, 9.2\% of patients experienced grade 4 to grade 5 permanent renal toxicity, $12.8 \%$ of patients developed transient grade 3 to 4 hematological toxicity, and 2 patients developed myelodysplastic syndrome and acute myeloid leukemia. ${ }^{94}$ Other studies including the NETTER-1 trial have reported lower rates of toxicities. ${ }^{96-99}$

\section{CONCLUSIONS}

While the mainstay for operable BPNETs is surgical resection, the role of different systemic therapies in the treatment of advanced/metastatic disease has evolved significantly over the years. We have summarized recommendations in a treatment algorithm (Figure 1). The timing of initiating treatment and choice and sequence of therapies is crucial. A multidisciplinary and patient-centered approach is necessary for optimizing a treatment plan. 


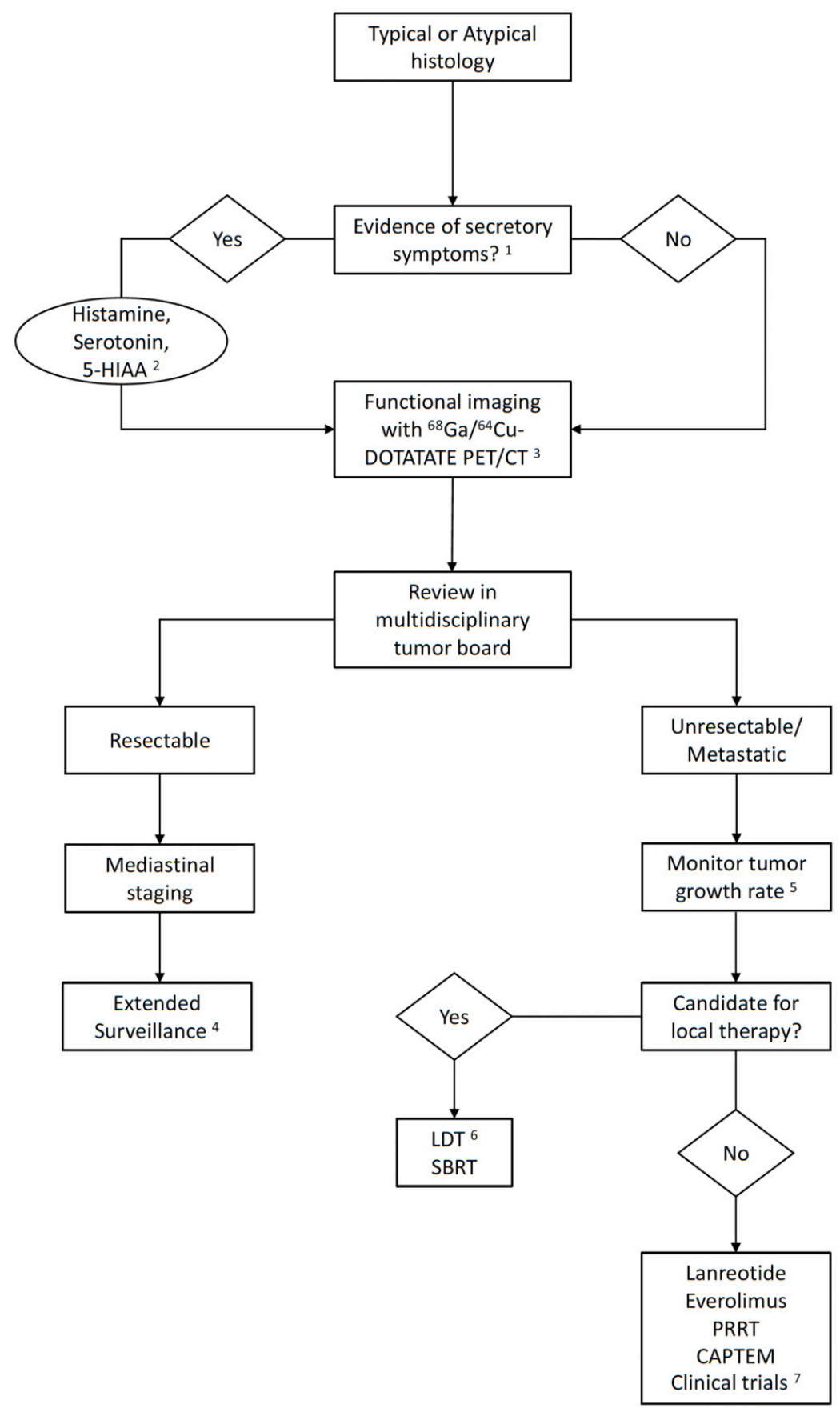

\section{Figure 1. Treatment algorithm for bronchopulmonary neuroendocrine tumors}

${ }^{1}$ Symptoms of carcinoid syndrome include cutaneous flushing, diarrhea and wheezing. Ectopic secretion of other hormones can result in acromegaly, hypoglycemia or cushing's syndrome.

2 Other tests such as CgA, ACTH, GHRH, Glucose, Insulin, Proinsulin, C-Peptide, IGF-1, IGF-2, Pro-IGF2 should be obtained based on clinical presentation. A trial of somatostatin analogues is warranted in patients in patients with carcinoid syndrome, especially when they are positive on SSTR functional imaging.

${ }^{3}$ Functional imaging includes SSTR scintigraphy and ${ }^{68} \mathrm{Ga}$ or ${ }^{64} \mathrm{Cu}$-DOTATATE PET/CT. PET/CT is preferred over scintigraphy when available.

${ }^{4}$ Prolonged follow-up post-surgical resection is recommended due to risk of late recurrences. Up to 10 year follow up is recommended. Recurrence risk is lowest in node negative typical carcinoids.

${ }^{5}$ For metastatic disease, tumor growth rate should be assessed based on serial imaging performed every 3-6 months. Treatment should be initiated at progression, uncontrolled/ worsening symptoms or high tumor burden (especially in the liver).

${ }^{6}$ Liver directed therapy includes surgical cytoreduction, ablation or embolization.

${ }^{7}$ Systemic therapy involves an individualized approach after a tumor board discussion, preferably at a neuroendocrine tumor center. For instance, in low burden, SSTR positive metastatic disease, SSA versus observation should be considered. For progressive disease, everolimus versus PRRT with ${ }^{177}$ Lu-DOTATATE should be considered. For high burden, symptomatic disease, chemotherapy with CAPTEM and local therapy should be considered. Clinical trials should be always be kept as a potential option and discussed with patients. Abbreviations: 5-HIAA, 5-hydroxyindoleacetic acid; ACTH, adrenocorticotropic hormone; CAPTEM, capecitabine temozolomide; CgA, chromogranin A; GHRH, growth hormone releasing hormone; IGF, insulin-growth factor; LDT, liver directed therapy; PET/CT, positron emission tomography/computed tomography; PRRT, peptide receptor radionuclide therapy; SBRT, stereotactic body radiation therapy; SSA, somatostatin analog; SSTR, somatostatin receptor 


\section{FUTURE DIRECTIONS}

Management of BPNETs has come a long way and has seen rapid advancements in our understanding of its molecular biology, histopathological classification, diagnostic modalities, and treatment options. The next big challenge for us is to define the efficacy of these novel agents in BPNET specific clinical trials and define treatment sequencing based on prospectively conducted studies with meaningful clinical endpoints. The rarity of BPNETs often poses enrollment challenges but a concerted effort between industry and oncology cooperative groups is the only way forward in pursuing meaningful and much needed clinical studies.

\section{Continuing Education Credit Information:}

The Binaytara Foundation is accredited by the Washington State Medical Association to provide continuing medical education for physicians.

The Binaytara Foundation designates this live activity for a maximum of 1.0 AMA PRA Category 1 Credit $^{\mathrm{TM}}$. Physicians should claim only the credit commensurate with the extent of their participation in the activity.

This activity meets the criteria for up to 1.0 hours of Category I CME credit to satisfy the relicensure requirements of the Washington State Medical Quality Assurance Commission.

Please click here to register and claim your CME credits.

Submitted: December 20, 2021 PST, Accepted: January 21, 2022 PST 


\section{REFERENCES}

1. Dasari A, Shen C, Halperin D, et al. Trends in the Incidence, Prevalence, and Survival Outcomes in Patients With Neuroendocrine Tumors in the United States. JAMA Oncol. 2017;3(10):1335-1342. doi:10.10 01/jamaoncol.2017.0589

2. Modlin IM, Lye KD, Kidd M. A 5-decade analysis of 13,715 carcinoid tumors. Cancer. 2003;97(4):934-959. doi:10.1002/cncr.11105

3. Pelosi G, Sonzogni A, Harari S, et al. Classification of pulmonary neuroendocrine tumors: new insights. Transl Lung Cancer Res. 2017;6(5):513-529. doi:10.21 $\underline{037 / \text { tlcr.2017.09.04 }}$

4. Travis WD, Brambilla E, Nicholson AG, et al. The 2015 World Health Organization Classification of Lung Tumors: Impact of Genetic, Clinical and Radiologic Advances Since the 2004 Classification. J Thorac Oncol. 2015;10(9):1243-1260. doi:10.1097/JT 0.0000000000000630

5. Fernandez-Cuesta L, McKay JD. Genomic architecture of lung cancers. Curr Opin Oncol. 2016;28(1):52-57. doi:10.1097/CCO.000000000000025 1

6. Rossi G, Cavazza A, Spagnolo P, et al. Diffuse idiopathic pulmonary neuroendocrine cell hyperplasia syndrome. Eur Respir J. 2016;47(6):1829-1841. doi:10.1183/13993003.0195 4-2015

7. Ferolla P, Daddi N, Urbani M, et al. Tumorlets, multicentric carcinoids, lymph-nodal metastases, and long-term behavior in bronchial carcinoids. J Thorac Oncol. 2009;4(3):383-387. doi:10.1097/JTO.0b013e318 $\underline{197 \mathrm{f} 2 \mathrm{e} 7}$

8. Gustafsson BI, Kidd M, Chan A, Malfertheiner MV, Modlin IM. Bronchopulmonary neuroendocrine tumors. Cancer. 2008;113(1):5-21. doi:10.1002/cncr.2 $\underline{3542}$

9. Naalsund A, Rostad H, Strom EH, Lund MB, Strand TE. Carcinoid lung tumors--incidence, treatment and outcomes: a population-based study. Eur J Cardiothorac Surg. 2011;39(4):565-569. doi:10.1016/ j.ejcts.2010.08.036

10. Daddi N, Ferolla P, Urbani M, et al. Surgical treatment of neuroendocrine tumors of the lung. Eur J Cardiothorac Surg. 2004;26(4):813-817. doi:10.1016/ j.ejcts.2004.05.052
11. Fink G, Krelbaum T, Yellin A, et al. Pulmonary carcinoid: presentation, diagnosis, and outcome in 142 cases in Israel and review of 640 cases from the literature. Chest. 2001;119(6):1647-1651. doi:10.137 8/chest.119.6.1647

12. Lim E, Yap YK, De Stavola BL, Nicholson AG, Goldstraw P. The impact of stage and cell type on the prognosis of pulmonary neuroendocrine tumors. $J$ Thorac Cardiovasc Surg. 2005;130(4):969-972. doi:1 $\underline{0.1016 / j . j t c v s .2005 .05 .041}$

13. Forde PM, Hooker CM, Boikos SA, et al. Systemic therapy, clinical outcomes, and overall survival in locally advanced or metastatic pulmonary carcinoid: a brief report. J Thorac Oncol. 2014;9(3):414-418. do i:10.1097/JTO.0000000000000065

14. Jeung MY, Gasser B, Gangi A, et al. Bronchial carcinoid tumors of the thorax: spectrum of radiologic findings. Radiographics. 2002;22(2):351-365. doi:10.1148/radiographics.22.2.g $\underline{02 \mathrm{mr} 01351}$

15. Melmon KL, Sjoerdsma A, Mason DT. Distinctive clinical and therapeutic aspects of the syndrome associated with bronchial carcinoid tumors. $\mathrm{Am} \mathrm{J}$ Med. 1965;39(4):568-581. doi:10.1016/0002-9343(6 5) $90080-\mathrm{x}$

16. Young J, Haissaguerre M, Viera-Pinto O, Chabre $\mathrm{O}$, Baudin E, Tabarin A. MANAGEMENT OF ENDOCRINE DISEASE: Cushing's syndrome due to ectopic ACTH secretion: an expert operational opinion. Eur J Endocrinol. 2020;182(4):R29-R58. doi:1 $\underline{0.1530 / E J E-19-0877}$

17. Krug S, Boch M, Rexin P, et al. Acromegaly in a patient with a pulmonary neuroendocrine tumor: case report and review of current literature. BMC Res Notes. 2016;9:326. doi:10.1186/s13104-016-2132-1

18. Shames JM, Dhurandhar NR, Blackard WG. Insulin-secreting bronchial carcinoid tumor with widespread metastases. Am J Med. 1968;44(4):632-637. doi:10.1016/0002-9343(68)9006 $\underline{5-X}$

19. Filosso PL, Kidd M, Roffinella M, et al. The utility of blood neuroendocrine gene transcript measurement in the diagnosis of bronchopulmonary neuroendocrine tumours and as a tool to evaluate surgical resection and disease progression. Eur J Cardiothorac Surg. 2018;53(3):631-639. doi:10.1093/ej cts/ezx386 
20. Vezzosi D, Walter T, Laplanche A, et al. Chromogranin A measurement in metastatic welldifferentiated gastroenteropancreatic neuroendocrine carcinoma: screening for false positives and a prospective follow-up study. Int J Biol Markers. 2011;26(2):94-101. doi:10.5301/JBM.2011.83 $\underline{27}$

21. Ramirez RA, Chauhan A, Gimenez J, Thomas $\mathrm{KEH}$, Kokodis I, Voros BA. Management of pulmonary neuroendocrine tumors. Rev Endocr Metab Disord. 2017;18(4):433-442. doi:10.1007/s11154-017-9429-9

22. Rosado de Christenson ML, Abbott GF, Kirejczyk WM, Galvin JR, Travis WD. Thoracic carcinoids: radiologic-pathologic correlation. Radiographics. 1999;19(3):707-736. doi:10.1148/radiographics.19.3.g 99ma11707

23. Chassagnon G, Favelle O, Marchand-Adam S, De Muret A, Revel MP. DIPNECH: when to suggest this diagnosis on CT. Clin Radiol. 2015;70(3):317-325. do i:10.1016/i.crad.2014.10.012

24. Vesterinen T, Leijon $\mathrm{H}$, Mustonen $\mathrm{H}$, et al. Somatostatin Receptor Expression Is Associated With Metastasis and Patient Outcome in Pulmonary Carcinoid Tumors. J Clin Endocrinol Metab. 2019;104(6):2083-2093. doi:10.1210/jc.2018-01931

25. Gabriel M, Decristoforo C, Kendler D, et al. 68GaDOTA-Tyr3-octreotide PET in neuroendocrine tumors: comparison with somatostatin receptor scintigraphy and CT. J Nucl Med. 2007;48(4):508-518. doi:10.2967/jnumed.106.035667

26. Jindal T, Kumar A, Venkitaraman B, et al. Evaluation of the role of [18F]FDG-PET/CT and [68Ga]DOTATOC-PET/CT in differentiating typical and atypical pulmonary carcinoids. Cancer Imaging. 2011;11:70-75. doi:10.1102/1470-7330.2011.0010

27. Venkitaraman B, Karunanithi S, Kumar A, Khilnani GC, Kumar R. Role of 68Ga-DOTATOC PET/ CT in initial evaluation of patients with suspected bronchopulmonary carcinoid. Eur J Nucl Med Mol Imaging. 2014;41(5):856-864. doi:10.1007/s00259-01 3-2659-5

28. Moore W, Freiberg E, Bishawi M, et al. FDG-PET imaging in patients with pulmonary carcinoid tumor. Clin Nucl Med. 2013;38(7):501-505. doi:10.1097/RL U.0b013e318279f0f5

29. Rami-Porta R, Asamura H, Travis WD, Rusch VW. Lung cancer - major changes in the American Joint Committee on Cancer eighth edition cancer staging manual. CA Cancer J Clin. 2017;67(2):138-155. doi:1 0.3322/caac. 21390
30. Filosso PL, Oliaro A, Ruffini E, et al. Outcome and prognostic factors in bronchial carcinoids: a singlecenter experience. $J$ Thorac Oncol.

2013;8(10):1282-1288. doi:10.1097/JTO.0b013e31829f $\underline{097 \mathrm{a}}$

31. Ramirez RA, Beyer DT, Diebold AE, et al. Prognostic Factors in Typical and Atypical Pulmonary Carcinoids. Ochsner J. 2017;17(4):335-340.

32. Pelosi G, Rodriguez J, Viale G, Rosai J. Typical and atypical pulmonary carcinoid tumor overdiagnosed as small-cell carcinoma on biopsy specimens: a major pitfall in the management of lung cancer patients. Am J Surg Pathol. 2005;29(2):179-187. doi:10.1097/0 1.pas.0000149690.75462.29

33. Singh S, Bergsland EK, Card CM, et al. Commonwealth Neuroendocrine Tumour Research Collaboration and the North American Neuroendocrine Tumor Society Guidelines for the Diagnosis and Management of Patients With Lung Neuroendocrine Tumors: An International Collaborative Endorsement and Update of the 2015 European Neuroendocrine Tumor Society Expert Consensus Guidelines. J Thorac Oncol. 2020;15(10):1577-1598. doi:10.1016/j.jtho.2020.06.02 1

34. Detterbeck FC. Management of carcinoid tumors. Ann Thorac Surg. 2010;89(3):998-1005. doi:10.1016/ j.athoracsur.2009.07.097

35. Brown LM, Cooke DT, Jett JR, David EA. Extent of Resection and Lymph Node Assessment for Clinical Stage T1aN0M0 Typical Carcinoid Tumors. Ann Thorac Surg. 2018;105(1):207-213. doi:10.1016/j.athor acsur.2017.07.049

36. Raz DJ, Nelson RA, Grannis FW, Kim JY. Natural history of typical pulmonary carcinoid tumors: a comparison of nonsurgical and surgical treatment. Chest. 2015;147(4):1111-1117. doi:10.1378/chest.14-1 $\underline{960}$

37. Cavaliere S, Foccoli P, Toninelli C. Curative Bronchoscopic Laser Therapy for Surgically Resectable Tracheobronchial Tumors: Personal Experience. Journal of Bronchology \& Interventional Pulmonology. 2002;9:90-95. doi:10.1097/00128594-20 0204000-00004

38. Sutedja TG, Schreurs AJ, Vanderschueren RG, Kwa B, vd Werf TS, Postmus PE. Bronchoscopic therapy in patients with intraluminal typical bronchial carcinoid. Chest. 1995;107(2):556-558. doi:10.1378/ch est.107.2.556 
39. Reuling E, Dickhoff C, Plaisier PW, et al. Endobronchial Treatment for Bronchial Carcinoid: Patient Selection and Predictors of Outcome. Respiration. 2018;95(4):220-227. doi:10.1159/0004849 $\underline{84}$

40. Guarino C, Mazzarella G, De Rosa N, et al. Presurgical bronchoscopic treatment for typical endobronchial carcinoids. Int J Surg. 2016;33 Suppl 1:S30-5. doi:10.1016/j.ijsu.2016.05.054

41. National Comprehensive Cancer Network Network. Neuroendocrine and Adrenal Tumors (Version 4.2021). National Comprehensive Cancer Network. Published 2021. https://www.nccn.org/profe ssionals/physician_gls/pdf/neuroendocrine.pdf

42. Baudin E, Caplin M, Garcia-Carbonero R, et al. Lung and thymic carcinoids: ESMO Clinical Practice Guidelines for diagnosis, treatment and follow-up. Annals of Oncology. 2021;32(4):439-451. doi:10.1016/ j.annonc.2021.01.003

43. Ramirez RA, Thomas K, Jacob A, Lin K, BrenMattison Y, Chauhan A. Adjuvant therapy for lung neuroendocrine neoplasms. World J Clin Oncol. 2021;12(8):664-674. doi:10.5306/wjco.v12.i8.664

44. Chong CR, Wirth LJ, Nishino M, et al. Chemotherapy for locally advanced and metastatic pulmonary carcinoid tumors. Lung Cancer. 2014;86(2):241-246. doi:10.1016/j.lungcan.2014.08.0 12

45. Herde RF, Kokeny KE, Reddy CB, et al. Primary Pulmonary Carcinoid Tumor: A Long-term Single Institution Experience. Am J Clin Oncol. 2018;41(1):24-29. doi:10.1097/COC.00000000000002 $\underline{21}$

46. Westin GFM, Alsidawi S, Leventakos K, Halfdanarson TR, Molina JR. Impact of adjuvant chemotherapy in non-metastatic node positive bronchial neuroendocrine tumors (BNET). Journal of Clinical Oncology. 2017;35(15_suppl):8533-8533. doi:1 0.1200/JCO.2017.35.15 suppl.8533

47. Caplin ME, Pavel M, Cwikla JB, et al. Lanreotide in metastatic enteropancreatic neuroendocrine tumors. N Engl J Med. 2014;371(3):224-233. doi:10.1056/NEJM oa1316158

48. Rinke A, Muller HH, Schade-Brittinger C, et al. Placebo-controlled, double-blind, prospective, randomized study on the effect of octreotide LAR in the control of tumor growth in patients with metastatic neuroendocrine midgut tumors: a report from the PROMID Study Group. J Clin Oncol. 2009;27(28):4656-4663. doi:10.1200/JCO.2009.22.851 $\underline{0}$
49. Caplin ME, Pavel M, Phan AT, et al. Lanreotide autogel/depot in advanced enteropancreatic neuroendocrine tumours: final results of the CLARINET open-label extension study. Endocrine. 2021;71(2):502-513. doi:10.1007/s12020-020-02475-2

50. Bongiovanni A, Recine F, Riva N, et al. Outcome Analysis of First-line Somatostatin Analog Treatment in Metastatic Pulmonary Neuroendocrine Tumors and Prognostic Significance of (18)FDG-PET/CT. Clin Lung Cancer. 2017;18(4):415-420. doi:10.1016/j.cllc.2 $\underline{016.11 .004}$

51. Lenotti E, Alberti A, Spada F, et al. Outcome of Patients With Metastatic Lung Neuroendocrine Tumors Submitted to First Line Monotherapy With Somatostatin Analogs. Front Endocrinol (Lausanne). 2021;12:669484. doi:10.3389/fendo.2021.669484

52. Sullivan I, Le Teuff G, Guigay J, et al. Antitumour activity of somatostatin analogues in sporadic, progressive, metastatic pulmonary carcinoids. Eur J Cancer. 2017;75:259-267. doi:10.1016/j.ejca.2016.11.0 $\underline{34}$

53. Baudin E, Horsch D, Singh S, et al. 10960 Lanreotide autogel/depot (LAN) in patients with advanced bronchopulmonary (BP) neuroendocrine tumors (NETs): Results from the phase III SPINET study. Annals of Oncology. 2021;32:S906. doi:10.1016/ j.annonc.2021.08.178

54. Lee L, Ito T, Jensen RT. Everolimus in the treatment of neuroendocrine tumors: efficacy, sideeffects, resistance, and factors affecting its place in the treatment sequence. Expert Opin Pharmacother. 2018;19(8):909-928. doi:10.1080/14656566.2018.1476 492

55. Scarpa A, Chang DK, Nones K, et al. Wholegenome landscape of pancreatic neuroendocrine tumours. Nature. 2017;543(7643):65-71. doi:10.1038/ nature21063

56. Zatelli MC, Minoia M, Martini C, et al. Everolimus as a new potential antiproliferative agent in aggressive human bronchial carcinoids. Endocr Relat Cancer. 2010;17(3):719-729. doi:10.1677/ERC-10-009 7

57. Yao JC, Shah MH, Ito T, et al. Everolimus for advanced pancreatic neuroendocrine tumors. $N$ Engl $J$ Med. 2011;364(6):514-523. doi:10.1056/NEJMoa10092 $\underline{90}$

58. Yao JC, Pavel M, Lombard-Bohas C, et al. Everolimus for the Treatment of Advanced Pancreatic Neuroendocrine Tumors: Overall Survival and Circulating Biomarkers From the Randomized, Phase III RADIANT-3 Study. J Clin Oncol. 2016;34(32):3906-3913. doi:10.1200/JCO.2016.68.070 $\underline{2}$ 
59. Pavel ME, Hainsworth JD, Baudin E, et al. Everolimus plus octreotide long-acting repeatable for the treatment of advanced neuroendocrine tumours associated with carcinoid syndrome (RADIANT-2): a randomised, placebo-controlled, phase 3 study. Lancet. 2011;378(9808):2005-2012. doi:10.1016/S014 $\underline{0-6736(11) 61742-X}$

60. Pavel ME, Baudin E, Oberg KE, et al. Efficacy of everolimus plus octreotide LAR in patients with advanced neuroendocrine tumor and carcinoid syndrome: final overall survival from the randomized, placebo-controlled phase 3 RADIANT-2 study. Ann Oncol. 2017;28(7):1569-1575. doi:10.1093/annonc/md $\underline{\mathrm{x} 193}$

61. Fazio N, Granberg D, Grossman A, et al. Everolimus plus octreotide long-acting repeatable in patients with advanced lung neuroendocrine tumors: analysis of the phase 3, randomized, placebocontrolled RADIANT-2 study. Chest. 2013;143(4):955-962. doi:10.1378/chest.12-1108

62. Yao JC, Fazio N, Singh S, et al. Everolimus for the treatment of advanced, non-functional neuroendocrine tumours of the lung or gastrointestinal tract (RADIANT-4): a randomised, placebo-controlled, phase 3 study. Lancet. 2016;387(10022):968-977. doi:10.1016/S0140-6736(1 5)00817-X

63. Fazio N, Buzzoni R, Delle Fave G, et al. Everolimus in advanced, progressive, welldifferentiated, non-functional neuroendocrine tumors: RADIANT-4 lung subgroup analysis. Cancer Sci. 2018;109(1):174-181. doi:10.1111/cas.13427

64. Bajetta E, Catena L, Fazio N, et al. Everolimus in combination with octreotide long-acting repeatable in a first-line setting for patients with neuroendocrine tumors: an ITMO group study. Cancer. 2014;120(16):2457-2463. doi:10.1002/cncr.28 $\underline{726}$

65. Bajetta E, Catena L, Pusceddu S, et al. Everolimus in Combination with Octreotide Long-Acting Repeatable in a First-Line Setting for Patients with Neuroendocrine Tumors: A 5-Year Update. Neuroendocrinology. 2018;106(4):307-311. doi:10.115 9/000479587

66. Ferolla P, Brizzi MP, Meyer T, et al. Efficacy and safety of long-acting pasireotide or everolimus alone or in combination in patients with advanced carcinoids of the lung and thymus (LUNA): an openlabel, multicentre, randomised, phase 2 trial. Lancet Oncol. 2017;18(12):1652-1664. doi:10.1016/S1470-20 45(17)30681-2
67. Raymond E, Dahan L, Raoul JL, et al. Sunitinib malate for the treatment of pancreatic neuroendocrine tumors. $N$ Engl J Med. 2011;364(6):501-513. doi:10.1056/NEJMoa1003825

68. Kulke MH, Lenz HJ, Meropol NJ, et al. Activity of sunitinib in patients with advanced neuroendocrine tumors. J Clin Oncol. 2008;26(20):3403-3410. doi:10.1 200/ICO.2007.15.9020

69. Grande E, Capdevila J, Castellano D, et al. Pazopanib in pretreated advanced neuroendocrine tumors: a phase II, open-label trial of the Spanish Task Force Group for Neuroendocrine Tumors (GETNE). Ann Oncol. 2015;26(9):1987-1993. doi:10.10 93/annonc/mdv252

70. Castellano D, Capdevila J, Sastre J, et al. Sorafenib and bevacizumab combination targeted therapy in advanced neuroendocrine tumour: a phase II study of Spanish Neuroendocrine Tumour Group (GETNE0801). Eur J Cancer. 2013;49(18):3780-3787. $\underline{\mathrm{d}}$ oi:10.1016/j.ejca.2013.06.042

71. Yao JC, Phan A, Hoff PM, et al. Targeting vascular endothelial growth factor in advanced carcinoid tumor: a random assignment phase II study of depot octreotide with bevacizumab and pegylated interferon alpha-2b. J Clin Oncol. 2008;26(8):1316-1323. doi:10.1200/JCO.2007.13.6374

72. Xu J, Shen L, Zhou Z, et al. Surufatinib in advanced extrapancreatic neuroendocrine tumours (SANET-ep): a randomised, double-blind, placebocontrolled, phase 3 study. Lancet Oncol. 2020;21(11):1500-1512. doi:10.1016/S1470-2045(20)3 $\underline{0496-4}$

73. Granberg D, Eriksson B, Wilander E, et al. Experience in treatment of metastatic pulmonary carcinoid tumors. Ann Oncol. 2001;12(10):1383-1391. doi:10.1023/a:1012569909313

74. Sun W, Lipsitz S, Catalano P, Mailliard JA, Haller DG, Eastern Cooperative Oncology G. Phase II/III study of doxorubicin with fluorouracil compared with streptozocin with fluorouracil or dacarbazine in the treatment of advanced carcinoid tumors: Eastern Cooperative Oncology Group Study E1281. J Clin Oncol. 2005;23(22):4897-4904. doi:10.1200/JCO.200 5.03.616

75. Turner NC, Strauss SJ, Sarker D, et al. Chemotherapy with 5-fluorouracil, cisplatin and streptozocin for neuroendocrine tumours. $\mathrm{Br} \mathrm{J}$ Cancer. 2010;102(7):1106-1112. doi:10.1038/sj.bjc.66 $\underline{05618}$ 
76. Meyer T, Qian W, Caplin ME, et al. Capecitabine and streptozocin $+/$ - cisplatin in advanced gastroenteropancreatic neuroendocrine tumours. Eur J Cancer. 2014;50(5):902-911. doi:10.1016/j.ejca.201 $\underline{3.12 .011}$

77. Masi G, Fornaro L, Cupini S, et al. Refractory neuroendocrine tumor-response to liposomal doxorubicin and capecitabine. Nat Rev Clin Oncol. 2009;6(11):670-674. doi:10.1038/nrclinonc.2009.148

78. Kulke MH, Wu B, Ryan DP, et al. A phase II trial of irinotecan and cisplatin in patients with metastatic neuroendocrine tumors. Dig Dis Sci. 2006;51(6):1033-1038. doi:10.1007/s10620-006-800 $\underline{1-3}$

79. Faure M, Niccoli P, Autret A, Cavaglione G, Mineur L, Raoul JL. Systemic chemotherapy with FOLFOX in metastatic grade $1 / 2$ neuroendocrine cancer. Mol Clin Oncol. 2017;6(1):44-48. doi:10.3892/ $\underline{\text { mco.2016.1097 }}$

80. Spada F, Antonuzzo L, Marconcini R, et al. Oxaliplatin-Based Chemotherapy in Advanced Neuroendocrine Tumors: Clinical Outcomes and Preliminary Correlation with Biological Factors. Neuroendocrinology. 2016;103(6):806-814. doi:10.115 9/000444087

81. Ferrarotto R, Testa L, Riechelmann RP, et al. Combination of Capecitabine and Oxaliplatin is an Effective Treatment Option for Advanced Neuroendocrine Tumors. Rare Tumors. 2013;5(3):e35. doi:10.4081/rt.2013.e35

82. Bajetta E, Catena L, Procopio G, et al. Are capecitabine and oxaliplatin (XELOX) suitable treatments for progressing low-grade and high-grade neuroendocrine tumours? Cancer Chemother Pharmacol. 2007;59(5):637-642. doi:10.1007/s00280-0 $\underline{\text { 06-0306-6 }}$

83. Walter T, Planchard D, Bouledrak K, et al. Evaluation of the combination of oxaliplatin and 5 -fluorouracil or gemcitabine in patients with sporadic metastatic pulmonary carcinoid tumors. Lung Cancer. 2016;96:68-73. doi:10.1016/j.lungcan.20 16.03.018

84. Brizzi MP, Berruti A, Ferrero A, et al. Continuous 5 -fluorouracil infusion plus long acting octreotide in advanced well-differentiated neuroendocrine carcinomas. A phase II trial of the Piemonte oncology network. BMC Cancer. 2009;9:388. doi:10.1186/147 1-2407-9-388

85. Crona J, Fanola I, Lindholm DP, et al. Effect of temozolomide in patients with metastatic bronchial carcinoids. Neuroendocrinology. 2013;98(2):151-155. d oi: $10.1159 / 000354760$
86. Ferolla P, Berruti A, Spada F, et al. 1161MO Lanreotide autogel (LAN) and temozolomide (TMZ) combination therapy in progressive thoracic neuroendocrine tumours (TNETs): ATLANT study results. Annals of Oncology. 2020;31:S773. doi:10.101 6/j.annonc.2020.08.1374

87. Chan JA, Stuart K, Earle CC, et al. Prospective study of bevacizumab plus temozolomide in patients with advanced neuroendocrine tumors. J Clin Oncol. 2012;30(24):2963-2968. doi:10.1200/ICO.2011.40.314 7

88. Ramirez RA, Beyer DT, Chauhan A, Boudreaux JP, Wang YZ, Woltering EA. The Role of Capecitabine/ Temozolomide in Metastatic Neuroendocrine Tumors. Oncologist. 2016;21(6):671-675. doi:10.1634/ theoncologist.2015-0470

89. Spada F, Antonuzzo L, Marconcini R, et al. Chemotherapy with capecitabine plus temozolomide (CAP-TEM) in patients with advanced neuroendocrine neoplasms (NENs): an Italian multicenter retrospective analysis. Journal of Clinical Oncology. 2015;33(15_suppl):e15174-e15174. doi:10.1 200/jco.2015.33.15_suppl.e15174

90. Thomas K, Voros BA, Meadows-Taylor M, et al. Outcomes of Capecitabine and Temozolomide (CAPTEM) in Advanced Neuroendocrine Neoplasms (NENs). Cancers (Basel). 2020;12(1). doi:10.3390/canc ers12010206

91. Al-Toubah T, Morse B, Strosberg J. Capecitabine and Temozolomide in Advanced Lung Neuroendocrine Neoplasms. Oncologist. 2020;25(1):e48-e52. doi:10.1634/theoncologist.201 9-0361

92. Fine RL, Gulati AP, Tsushima D, et al. Prospective phase II study of capecitabine and temozolomide (CAPTEM) for progressive, moderately, and welldifferentiated metastatic neuroendocrine tumors. Journal of Clinical Oncology. 2014;32(3_suppl):179-179. doi:10.1200/jco.2014.3 2.3 suppl.179

93. Waldherr C, Pless M, Maecke HR, Haldemann A, Mueller-Brand J. The clinical value of [90Y-DOTA]-DPhe1-Tyr3-octreotide (90Y-DOTATOC) in the treatment of neuroendocrine tumours: a clinical phase II study. Ann Oncol. 2001;12(7):941-945. doi:1 0.1023/a:1011160913619

94. Imhof A, Brunner P, Marincek N, et al. Response, Survival, and Long-Term Toxicity After Therapy With the Radiolabeled Somatostatin Analogue [90YDOTA]-TOC in Metastasized Neuroendocrine Cancers. Journal of Clinical Oncology. 2011;29(17):2416-2423. doi:10.1200/jco.2010.33.7873 
95. Sharma N, Naraev BG, Engelman EG, et al. Peptide Receptor Radionuclide Therapy Outcomes in a North American Cohort With Metastatic WellDifferentiated Neuroendocrine Tumors. Pancreas. 2017;46(2):151-156. doi:10.1097/MPA.000000000000 $\underline{0734}$

96. Mariniello A, Bodei L, Tinelli C, et al. Long-term results of PRRT in advanced bronchopulmonary carcinoid. Eur J Nucl Med Mol Imaging.

2016;43(3):441-452. doi:10.1007/s00259-015-3190-7

97. Parghane RV, Talole S, Prabhash K, Basu S. Clinical Response Profile of Metastatic/Advanced Pulmonary Neuroendocrine Tumors to Peptide Receptor Radionuclide Therapy with $177 \mathrm{Lu}-$ DOTATATE. Clin Nucl Med. 2017;42(6):428-435. doi:1 0.1097/RLU.0000000000001639
98. Sabet A, Haug AR, Eiden C, et al. Efficacy of peptide receptor radionuclide therapy with (177)Luoctreotate in metastatic pulmonary neuroendocrine tumors: a dual-centre analysis. Am J Nucl Med Mol Imaging. 2017;7(2):74-83.

99. Strosberg J, El-Haddad G, Wolin E, et al. Phase 3 Trial of (177)Lu-Dotatate for Midgut Neuroendocrine Tumors. N Engl J Med. 2017;376(2):125-135. doi:10.10 56/NEJMoa1607427

100. Strosberg JR, Caplin ME, Kunz PL, et al. Final overall survival in the phase 3 NETTER-1 study of lutetium-177-DOTATATE in patients with midgut neuroendocrine tumors. Journal of Clinical Oncology. 2021;39(15_suppl):4112-4112. doi:10.1200/JCO.202 1.39.15_suppl.4112 